Mortalin Biology: Life, Stress and Death 
Sunil C. Kaul • Renu Wadhwa

Editors

\section{Mortalin Biology: Life, Stress and Death}

然 Springer 


\section{Editors}

Sunil C. Kaul

National Institute of Advanced Industrial Science and Technology

Tsukuba, Ibaraki

Japan

\section{Renu Wadhwa}

National Institute of Advanced Industrial Science and Technology (AIST)

Tsukuba, Ibaraki

Japan

ISBN 978-94-007-3026-7

e-ISBN 978-94-007-3027-4

DOI 10.1007/978-94-007-3027-4

Springer Dordrecht Heidelberg London New York

Library of Congress Control Number: 2012933850

(C) Springer Science+Business Media B.V. 2012

No part of this work may be reproduced, stored in a retrieval system, or transmitted in any form or by any means, electronic, mechanical, photocopying, microfilming, recording or otherwise, without written permission from the Publisher, with the exception of any material supplied specifically for the purpose of being entered and executed on a computer system, for exclusive use by the purchaser of the work.

Printed on acid-free paper

Springer is part of Springer Science+Business Media (www.springer.com) 


\section{Foreword}

The heat shock response was originally discovered over 40 years ago in the fruit fly and heat shock proteins were subsequently identified in all organisms. It was found that these protect organisms against exposure to suboptimal temperatures and numerous other stresses many of which are also inducers of the response. Research on the mechanisms of action of these stress proteins was aided by development of molecular technologies, identification and cloning of new genes encoding stress proteins in a variety of assay systems both at the cellular and organism levels and it became clear that these proteins have essential constitutive functions in normal unstressed conditions. While in vivo animal systems are necessary to make certain firm conclusions, culture cell systems still offer an excellent platform to dissect the molecular mechanisms of action of a protein and to uncover the signal transduction pathways involved in the response. In my view, mortalin is an excellent example of this. Besides its cloning in the normal and cancer cell hybrid screening assay, many of its characteristics such as multiple subcellular residences, impact on p53 protein activity and carcinogenesis, and involvement in neuro-degenerative pathologies have been found since its discovery. The present book offers a single volume reading on the discovery of mortalin biology by experts from different fields and different parts of the globe. It is a unique volume compiling structural, evolutionary and functional aspects of a single stress protein in a variety of model systems ranging from invertebrates to human cells in culture and clinical samples. Besides making an easily understandable reading, it will be very helpful in asking further questions and designing experiments to advance mortalin-based diagnostics and therapeutics.

IBIS, Pavillon C.E. Marchand

1030 Ave de la médecine

Université Laval

Québec, Qc, Canada G1V 0A6

Phone: 418 656-3339

Fax 418 656-7176

E-mail: robert.tanguay@ibis.ulaval.ca
Robert M. Tanguay, D.Sc. Professor and Associate Head

Dept. Molecular Biology Medical Biochemistry \& Pathology Lab Cell \& Developmental Genetics 


\section{Preface}

Since the discovery of heat shock response by Ferruccio Ritossa in 1962, the phenomenon has been well characterized in a variety of cells and organisms as induction of a family of proteins called "heat shock proteins" (HSP). Based on their molecular weight, these proteins are classified into, at least, 6 major subfamilies named as HSP100, HSP90, HSP70, HSP60, HSP40 and small HSPs. The fact that the heat shock protein synthesis can be triggered by a variety of other stress conditions such as, infection, inflammation, exercise, starvation, oxygen-, nitrogen- or water-deprivation and exposure to chemical and physical toxins, they are also classified as "stress proteins". Then came the surprise that the HSP also exist under non-stressful conditions and perform housekeeping functions, such as folding and assisting in the establishment of correct protein conformation, mediating proteinprotein interactions, intra-cellular trafficking of other proteins, preventing unwanted protein aggregation and channelizing their degradation. A new term "chaperones" evolved to express such functionality of this highly conserved class of proteins.

A new member of HSP70 family of proteins was first cloned in 1993 in a cell hybrid protein-screening assay. Since it was identified to be associated with cellular mortal phenotype, it was named 'mortalin'. Endorsing its multiple functionality, mortalin made its manifestation in many independent experimental regimes, such as those aimed to identify molecules involved in antigen processing, stress-survival and mitochondrial functions. With nearly two decades of experimentation, mortalin has been recognized as an essential protein that not only acts as a chaperone and stress-survival factor but also plays a key role in mitochondrial import motor function, energy generation, ROS management, immune response, control of centrosome duplication and activities of tumor suppressor protein $\mathrm{p} 53$. Stemming from these multiple functions is its role in human cancers on one-hand and neurodegenerative diseases on the other. With an aim to introduce mortalin at the graduate and advanced undergraduate levels, this book is organized as a chapter-wise description of structure, evolution and functional role of mortalin in normal and diseased physiology. We hope that this sketch of mortalin biology by the team of experts will help in asking new questions, advancing knowledge and developing mortalin-based diagnostic and therapeutic reagents and technologies. 
We are very grateful to all the authors for their interest, enthusiasm and devotion to mortalin research that made this book necessary and possible. Without their hard work to contribute chapters, it was not possible to accomplish this volume suitable for general and specialized reading.

Sunil C. Kaul

Renu Wadhwa 


\section{Contents}

\section{Part I Structure and Function of Mortalin}

1 Birth of Mortalin: Multiple Names, Niches and Functions Connecting Stress, Senescence and Cancer

Renu Wadhwa and Sunil C. Kaul

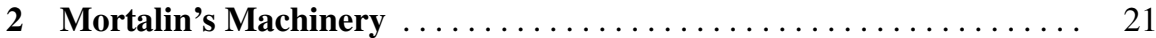

Custer C. Deocaris, Sunil C. Kaul and Renu Wadhwa

3 The Role of Mortalin in Iron Homeostasis . . . . . . . . . . . . . 31

Wen-I Luo and James A. Cowan

4 Functional Characteristics of Mortalin $\ldots \ldots \ldots \ldots \ldots \ldots \ldots \ldots \ldots$

Walter A. Baseler, Tara L. Croston and John M. Hollander

\section{Part II Mortalin in Evolution}

5 Mortalin and Drosophila DmHsp22: Two Mitochondrial Chaperones Regulating Aging and Carcinogenesis

Marie Le Pécheur, Geneviève Morrow and Robert M. Tanguay

6 Mortalin in Invertebrates and The Induction of Apoptosis

by Wild-Type p53 Following Defeat of Mortalin-Based

Cytoplasmic Sequestration in Cancerous Clam Hemocytes

Charles W. Walker, Ben Low and S. Anne Böttger

7 Mortalin and Stem Cells: A Study from Planarians

Renata Batistoni

Part III Mortalin in Health and Disease

8 Mortalin in Cell Protection from Immune Attack Moran Saar, Oren Moskovich and Zvi Fishelson

9 Mortalin in Neurological Diseases

Jinghua Jin, Travis J. Cook, Jake G. Hoekstra and Jing Zhang 
10 Loss of Mortalin Function in Parkinson's Disease-Supporting the Mitochondrial Pathway of Neurodegeneration

Lena F. Burbulla and Rejko Krüger

11 Hsp75/mortalin and Protection from Ischemic Brain Injury . . . . . . . 179 Robin E. White, Yi-Bing Ouyang and Rona G. Giffard

12 Catecholamine Regulated Protein (CRP40), A Splice Variant of Mortalin-2: Functional Role in CNS Disorders . . . . . . . . . . . . . 191 Joseph P. Gabriele, Sarah E. Groleau, Ritesh P. Daya, Zdenek B. Pristupa and Ram K. Mishra

13 Chaperonopathies: Diseases in Which Mortalin and Other Hsp-Chaperones Play a Role in Etiology and Pathogenesis . . . . . . . . . 209 Alberto J. L. Macario, Francesco Cappello and Everly Conway de Macario

\section{Part IV Mortalin and Cancer}

14 Many Faces of Mortalin and Tid1

Ohad Iosefson and Abdussalam Azem

15 Mortalin: A Positive Regulator of Centrosome Duplication and Amplification Masayuki Kanai and Kenji Fukasawa

16 Mortalin Expression in Normal and Neoplastic Tissues Angheliki Nomikos, Sinclair R. Dundas and Graeme I. Murray

17 Mortalin-p53 Interaction as a Target for Liver Cancer Therapy . . . . 267 Wen-Jing Lu, Nikki P. Lee, Renu Wadhwa and John M. Luk

18 Mortalin Targeting Gadgets for Cancer Therapy . . . . . . . . . . . . . 279 Chae-Ok Yun and Renu Wadhwa

\section{Part V Mortalin Based Technologies}

19 Cell Internalizing Anti-Mortalin Antibody for Generation of Illuminating MSCs for Long-Term In vitro and In vivo Tracking . . 295 Toshimasa Uemura, Masanori Nishi, Sunil C. Kaul and Renu Wadhwa

20 Mortalin Staining Pattern as a Reporter for Cell Based Anti-Cancer Drug Screening . . . . . . . . . . . . . . . . . . . . . . . 307 Ran Gao, Zeenia Kaul, Tomoko Yaguchi and Renu Wadhwa

21 Cell Internalizing Anti-mortalin Antibody as a Nanocarrier . . . . . . . 323 Zeenia Kaul, Tomoko Yaguchi, Renu Wadhwa and Sunil C. Kaul 


\section{Contributors}

Abdussalam Azem Department of Biochemistry and Molecular Biology, George S. Wise Faculty of Life Sciences, Tel Aviv University, Tel Aviv 69978, Israel

Walter A. Baseler West Virginia University School of Medicine, Division of Exercise Physiology, Center for Cardiovascular and Respiratory Sciences, 1 Medical Center Drive Morgantown, WV 26506, USA

Renata Batistoni Dipartimento di Biologia, Unità di Biologia Cellulare e dello Sviluppo, Università di Pisa. S.S.12 Abetone e Brennero 4, 56127 Pisa, Italy

S. Anne Böttger Department of Biology, West Chester University, West Chester, Pennsylvania 19383, USA

Lena F. Burbulla Laboratory of Functional Neurogenomics, Center of Neurology, Hertie-Institute for Clinical Brain Research and German Center for Neurodegenerative Diseases (DZNE), University of Tübingen, Hoppe-Seyler-Str. 3, 72076 Tübingen, Germany

Francesco Cappello IEMEST, Istituto Euro-Mediterraneo di Scienza e Tecnologia, Palermo, Italy

Travis J. Cook Department of Environmental \& Occupational Health Sciences, University of Washington School of Public Health, Seattle, WA 98195, USA

James A. Cowan Evans Laboratory of Chemistry, The Ohio State University, 100 West 18th Avenue, Columbus, Ohio 43210, USA

Tara L. Croston West Virginia University School of Medicine, Division of Exercise Physiology, Center for Cardiovascular and Respiratory Sciences, 1 Medical Center Drive Morgantown, WV 26506, USA

Ritesh P. Daya McMaster University, 1200 Main St. West, HSC 4N81, Hamilton, Ontario, L8N 3Z5, Canada

Custer C. Deocaris Department of Food Science and Technology, College of Home Economics, University of the Philippines, Diliman, Quezon City, Philippines 
Sinclair R. Dundas Department of Pathology, University Medical Buildings, Foresterhill, Aberdeen, AB25 2ZD, Scotland, United Kingdom

Zvi Fishelson Department of Cell and Developmental Biology, Sackler School of Medicine, Tel Aviv University, Tel Aviv 69978, Israel

Kenji Fukasawa Molecular Oncology Program, H. Lee Moffitt Cancer Center and Research Institute, Tampa, FL 33612, USA

Ran Gao National Institute of Advanced Industrial Science \& Technology (AIST), Central 4, 1-1-1 Higashi, Tsukuba, Ibaraki 305-8562, Japan

Joseph P. Gabriele McMaster University, 1200 Main St. West, HSC 4N81, Hamilton, Ontario, L8N 3Z5, Canada

Rona G. Giffard Dept. of Anesthesia, Stanford University School of Medicine, 300 Pasteur Drive, S272 Grant Building, CA 94305-5117, USA

Sarah E. Groleau McMaster University, 1200 Main St. West, HSC 4N81, Hamilton, Ontario, L8N 3Z5, Canada

Jake G. Hoekstra Department of Pathology, University of Washington School of Medicine, Seattle, WA 98104, USA

John M. Hollander West Virginia University School of Medicine, Division of Exercise Physiology, Center for Cardiovascular and Respiratory Sciences, 1 Medical Center Drive Morgantown, WV 26506, USA

Ohad Iosefson Department of Biochemistry and Molecular Biology, George S. Wise Faculty of Life Sciences, Tel Aviv University, Tel Aviv 69978, Israel

Jinghua Jin Department of Neurobiology, Zhejiang University School of Medicine, Hangzhou, Zhejiang 310058, China

Masayuki Kanai Molecular Oncology Program, H. Lee Moffitt Cancer Center and Research Institute, Tampa, FL 33612, USA

Sunil C. Kaul National Institute of Advanced Industrial Science \& Technology (AIST), Central 4, 1-1-1 Higashi, Tsukuba, Ibaraki 305-8562, Japan

Zeenia Kaul Center for Childhood Cancer, The Research Institute at Nationwide Children's Hospital, Columbus, Ohio, USA

Rejko Krüger Laboratory of Functional Neurogenomics, Center of Neurology, Hertie-Institute for Clinical Brain Research and German Center for Neurodegenerative Diseases (DZNE), University of Tübingen, Hoppe-Seyler-Str. 3, 72076 Tübingen, Germany

Nikki P. Lee Department of Surgery, Li Ka Shing Faculty of Medicine, The University of Hong Kong, Hong Kong, China

Ben Low The Jackson Laboratory, 600 Main Street, Bar Harbor, Maine 04609, USA 
Wen-Jing Lu Department of Surgery, Li Ka Shing Faculty of Medicine, The University of Hong Kong, Hong Kong, China

John M. Luk Department of Oncology, Roche R\&D Center (China) Ltd., 720 Cai Lun Road, Shanghai 201203, China

Wen-I Luo Ohio State Biochemistry Program, The Ohio State University, 100 West 18th Avenue, Columbus, OH 43210, USA

Alberto J. L. Macario Department of Microbiology and Immunology, School of Medicine, University of Maryland at Baltimore; and IMET, Columbus Center, 701 East Pratt Street, Baltimore, Maryland 21202, USA

Everly Conway de Macario Department of Microbiology and Immunology, School of Medicine, IMET, University of Maryland at Baltimore, Columbus Center, 701 East Pratt Street, Baltimore, Maryland 21202, USA

Ram K. Mishra McMaster University, 1200 Main St. West, HSC 4N81, Hamilton, Ontario, L8N 3Z5, Canada

Geneviève Morrow Laboratory of Cell and Developmental Genetics, Department of Molecular Biology, Medical Biochemistry and Pathology, Institut de Biologie Intégrative et des Systèmes and PROTEO, Université Laval, Québec, G1V 0A6, Canada

Oren Moskovich Department of Cell and Developmental Biology, Sackler School of Medicine, Tel Aviv University, Tel Aviv 69978, Israel

Graeme I. Murray Department of Pathology, University Medical Buildings, Foresterhill, Aberdeen, AB25 2ZD, Scotland, United Kingdom

Masanori Nishi National Institute of Advanced Industrial Science \& Technology (AIST), 1-1-1 Higashi, Tsukuba, Ibaraki 305-8562, Japan

Angheliki Nomikos Department of Pathology, University Medical Buildings, Foresterhill, Aberdeen, AB25 2ZD, Scotland, United Kingdom

Yi-Bing Ouyang Dept. of Anesthesia, Stanford University School of Medicine, 300 Pasteur Drive, S272 Grant Building, CA 94305-5117, USA

Marie Le Pécheur Laboratory of Cell and Developmental Genetics, Department of Molecular Biology, Medical Biochemistry and Pathology, Institut de Biologie Intégrative et des Systèmes and PROTEO, Université Laval, Québec, G1V 0A6, Canada

Zdenek B. Pristupa McMaster University, 1200 Main St. West, HSC 4N81, Hamilton, Ontario, L8N 3Z5, Canada

Moran Saar Department of Cell and Developmental Biology, Sackler School of Medicine, Tel Aviv University, Tel Aviv 69978, Israel 
Robert M. Tanguay Laboratory of Cell and Developmental Genetics, Department of Molecular Biology, Medical Biochemistry and Pathology, Institut de Biologie Intégrative et des Systèmes and PROTEO, Université Laval, Québec, G1V 0A6, Canada

Toshimasa Uemura National Institute of Advanced Industrial Science \& Technology (AIST), 1-1-1 Higashi, Tsukuba, Ibaraki 305-8562, Japan

Renu Wadhwa National Institute of Advanced Industrial Science \& Technology (AIST), Central 4, 1-1-1 Higashi, Tsukuba, Ibaraki 305-8562, Japan

Charles W. Walker Department of Molecular, Cellular and Biomedical Sciences, Center For Marine Biology and Marine Biomedical Research Group, The University of New Hampshire, Durham, NH 03824, USA

Robin E. White Dept. of Anesthesia, Stanford University School of Medicine, 300 Pasteur Drive, S272 Grant Building, CA 94305-5117, USA

Tomoko Yaguchi National Institute of Advanced Industrial Science \& Technology (AIST), Central 4, 1-1-1 Higashi, Tsukuba, Ibaraki 305-8562, Japan

Chae-Ok Yun Department of Bioengineering, College of Engineering, Hanyang University, 17 Haengdang-Dong, Seongdong-Gu, Seoul 133-791, Korea

Jing Zhang Department of Pathology, University of Washington School of Medicine, Seattle, WA 98104, USA 